\title{
The Deceptive Myxoid Appearance of a Low-Grade Cardiac Sarcoma and Its Accompanying Diagnostic Challenges: A Case Report
}

\author{
Kambiz Mozaffari, ${ }^{1}$ Hooman Bakhshandeh,,${ }^{2,}$ Reza Kiani, ${ }^{3}$ Nader Givtaj, ${ }^{4}$ and Hamid Reza Pooraliakbr ${ }^{5}$ \\ ${ }^{1}$ Associate Professor of Pathology, Rajaie Cardiovascular Medical and Research Center, Iran University of Medical Sciences, Tehran, IR Iran \\ ${ }^{2}$ Associate Professor of Epidemiology, Rajaie Cardiovascular Medical and Research Center, Iran University of Medical Sciences, Tehran, IR Iran \\ ${ }^{3}$ Associate Professor of Cardiology, Cardiac Intervention Research Center, Rajaie Cardiovascular Medical and Research Center, Iran University of Medical Sciences, Tehran, IR \\ Iran \\ ${ }^{4}$ Associate Professor of Cardiac Surgery, Rajaie Cardiovascular Medical and Research Center, Iran University of Medical Sciences, Tehran, IR Iran \\ ${ }^{5}$ Assistant Professor of Radiology, Rajaie Cardiovascular Medical and Research Center, Iran University of Medical Sciences, Tehran, IR Iran \\ "Corresponding author: Hooman Bakhshandeh, Rajaie Cardiovascular, Medical and Research Center, Mellat Park, Vali Asr Ave., Tehran, IR Iran. Tel: +98-2123923138, Fax: \\ +98-2122666317, E-mail: hooman.bakhshande@gmail.com
}

Received 2016 November 15; Accepted 2016 December 24.

\begin{abstract}
We present a 40-year-old man with a recurrent left atrial mass, previously diagnosed as cardiac myxoma elsewhere. His new admission was due to a regrowth of the mass. Progressive exertional dyspnea was his major complaint. A large lobulated tumor was seen in echocardiography in the posterior wall of left atrium involving the posterior leaflet of mitral valve, and resulting in severe stenosis and diastolic protrusion of the mass into the left ventricle. Surgery showed a non-homogenous myxomatous mass which infiltrated the posterior wall of the left atrium and parts of the inter-atrial septum. As a result, the surgeon excised parts of the septum and the posterior wall and did a reconstruction with pericardial patches. The pathologic examination revealed soft creamy-brown tumoral fragments, $\mathrm{m}: 10 \times 4 \mathrm{~cm}$ altogether. Contrary to his previous diagnosis, we observed a malignant neoplasm with cells that had plump, round to oval nuclei, set in a myxoid and vascular background, but mitotic figures and necrosis were not conspicuous. Therefore, we made a diagnosis of recurrent myxoid round cell sarcoma and recommended immunohistochemical (IHC) studies. Multiple IHC and histopathological studies elsewhere favored a diagnosis of myxoma which was not compatible with our diagnosis. A second recurrent mass was soon found in the left atrium making further surgical attempts impossible. At last, a diagnosis of myxoid leiomyosarcoma confirmed our diagnosis, but the patient was not fortunate enough to survive and passed away before heart transplantation could be done as a remedy.
\end{abstract}

Keywords: Heart Neoplasms, Rare Diseases, Leiomyosarcoma, Recurrence

\section{Introduction}

Primary heart tumors are rare, whereas metastases are more common. Myxoma is the most prevalent primary neoplasia in the heart. Here, we discuss a rare and enigmatic case, initially presented with a deceptive appearance of a myxoma. The aim of this case report is to alert the cardiologists, cardiac surgeons, and pathologists, familiarizing them with the diagnostic pitfalls. Echocardiography, the onset of symptoms, and the location of the tumor, all serve as clues to a better approach (1).

\section{Case Presentation}

We present a 40-year-old man who had undergone a cardiac surgery in Ahvaz, one year prior to his admission in our center. A left atrial mass with a histopathological diagnosis of myxoma had been removed. According to the pathology report reviewed here later, the mass was a gray and myxoid lobulated tumor, m: $8 \times 6 \times 5.5 \mathrm{~cm}$. Therefore, his new admission was in fact the consequence of a regrowth of the mass, as a result of which, he had developed progressive exertional dyspnea and a repeat evaluation showed the recurrence of his left atrial mass.

The physical examination revealed a systolic murmur (grade 3/6) in apical area with radiation to axilla. Electrocardiogram was normal. The patient underwent a thorough trans-thoracic and trans-esophageal echocardiographic evaluation which showed a large lobulated tumor, attached to the posterior wall of left atrium. The posterior leaflet of the mitral valve was involved by the cardiac mass, resulting in severe stenosis of the ventricular inlet (gradient) and diastolic protrusion of the mass into the left ventricle (Figure 1).

Other echocardiographic findings such as chamber sizes, left ventricular function, aortic and tricuspid valve morphology and function were normal. There was no evidence of another mass in any other cardiac chambers.

The patient was referred to surgery for a repeat surgical excision. Exploration in the operating room showed a non-homogenous myxomatous mass which infiltrated the 


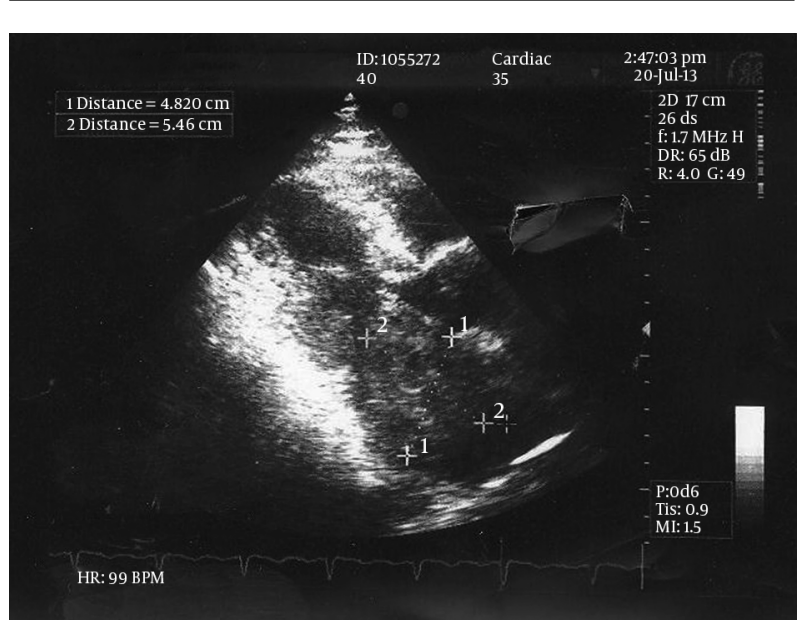

$\overline{\text { Figure 1. Left Atrial Cardiac Mass, Resulting in Severe Stenosis of the Ventricular Inlet }}$ and Diastolic Protrusion into the Left Ventricle

posterior wall of the left atrium and parts of the inter-atrial septum. As a result, the surgeon excised parts of the septum and the posterior wall and did a reconstruction of the area with pericardial patches. The pathologic examination of the recurrent mass was as follows: soft creamy-brown tumoral fragments, m: 10x4 cm altogether. Contrary to his previous diagnosis, we observed a malignant neoplasm with cells that had plump, round to oval nuclei. The cells were set in a myxoid and vascular background, but mitotic figures and necrosis were not conspicuous. Based on the above findings, we made a diagnosis of recurrent myxoid round cell sarcoma and recommended immunohistochemical (IHC) studies in order to determine the nature of the tumor. The paraffin-embedded blocks of the first mass which was excised in Ahvaz were sent to another laboratory in that city. Again, the first diagnosis of myxoma was confirmed there by the pathologist. This was in contrast to our diagnosis of sarcoma. At his family's instigation our paraffin-embedded blocks were recut and examined by another pathology laboratory in Tehran. This time, a loose myxoid matrix with very sparse cellularity was noted. The cells were reported to have prominent spindle-shaped nuclei with high nucleocytoplasmic ratio, but no mitosis or necrosis was seen. Overall, the pathologist's diagnosis on our paraffin-embedded blocks was consistent with recurrent cardiac myxoma. Now faced with a dilemma, we decided to have all the reports and specimens from Ahvaz and Tehran seen and examined by another pathology laboratory in Tehran, where IHC study could be performed as well. The report this time came as follows: a neoformed tissue composed of spindle cells with elongated bland nuclei and amphophilic cytoplasm scattered in an extensively myxoid background. Foci of increased cellularity around vascular channels were reported. The IHC study was performed on paraffin-embedded blocks. The tumoral cells were positive for vimentin and smooth muscle actin, but were negative for Desmin, H-Caldesmon, CD99, and Calretinin. The final diagnosis was compatible with myxoma.

After surgery an oncologic consultation was requested. A complete CT scan of the thoraco-abdominal area showed no evidence of metastatic tumor in the liver, mediastinum and any other organs. According to the oncologist, chemotherapy or radiotherapy of the region was not effective due to the low grade of the cardiac malignancy and such treatments were not considered for the patient. Four months later, the patient returned with dyspnea and a large left atrial mass with obstructive effect on the mitral valve. This time a cardiac MRI with gadolinium was performed and revealed involvement of the ostium of the right upper and lower pulmonic veins by the tumor. After a repetitive surgical consultation it was decided that the patient be referred to a heart transplantation unit, inasmuch as further reconstruction of the area was nearly impossible. The patient was put on heart transplant waiting list, but unfortunately died one month later, before an appropriate donor could be found.

Obviously, there was a second recurrence. This time, our paraffin-embedded blocks from the second operation were again reviewed in another laboratory with a second IHC study, where positive staining with smooth muscle actin was noted, whereas s-100 was negative. Based on the histopathology and IHC findings, at last a diagnosis of lowgrade leiomyosarcoma was established, which confirmed our diagnosis. The second recurrence did not allow an excisional attempt and the patient passed away in another center with complications of superior vena cava syndrome (Figures 2-5).

\section{Discussion}

Primary heart tumors are frankly rare and, myxomas constitute some $80 \%$ of these primary neoplasms. Some myxoid neoplasms, either benign or malignant, may be mistaken for benign myxomas. Myxosarcomas for example, are fibroblastic or fibrohistiocytic in nature, and depict an extensively myxoid stroma; however, they must not be construed as benign myxomas that have evolved into sarcomas. In fact, there is little proof, attesting that myxomas possess such a progressive malignant transformation (1-3).

Around $80 \%$ of such sarcomas affect the left atrium, so the significance of a careful differential diagnosis from myxomas can be felt. A large number of cardiac myxoid sarcomas are initially misdiagnosed as myxomas, how- 


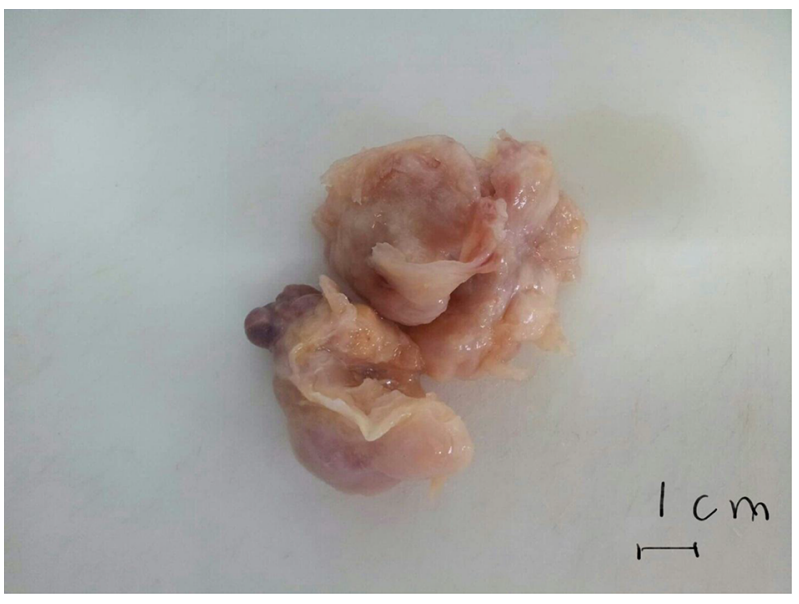

Figure 2. Soft Creamy-Brown Tumoral Fragments, $\mathrm{m}: 10 \times 4 \mathrm{~cm}$ Altogether

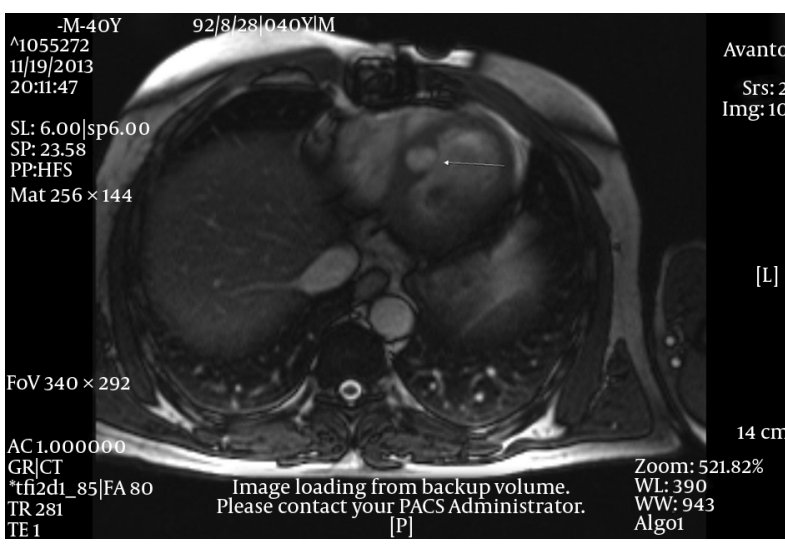

Figure 3. Figure 3

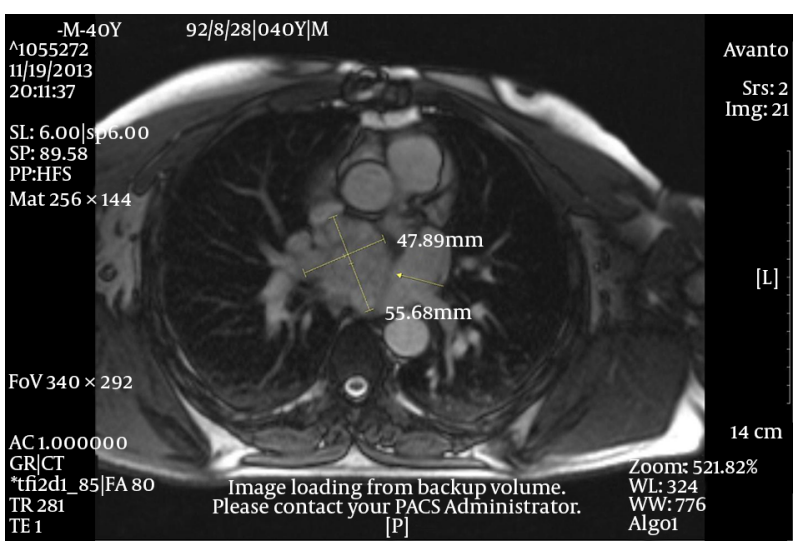

Figure 4. Figure 4

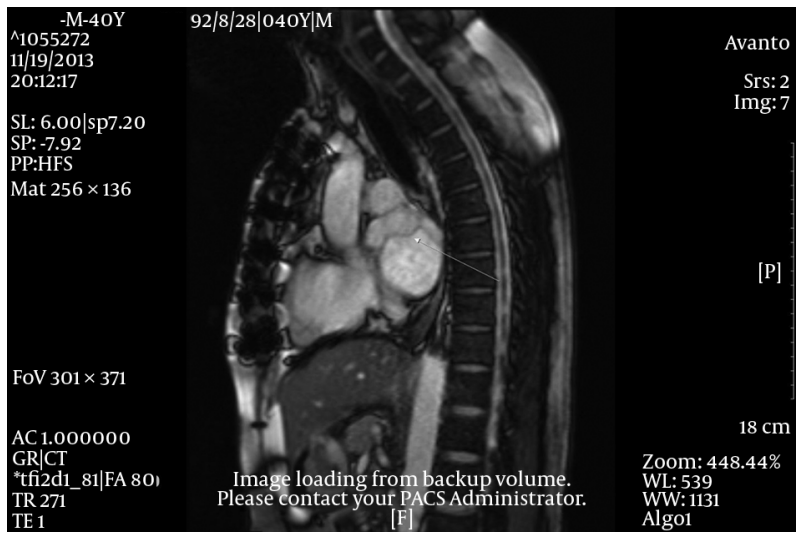

Figure 5. Figure 5

ever, the clues which aid to differentiate them from myxomas include myxoma or stellate cells, hemosiderin-laden macrophages, and factor-XIII-positive dendritic cells. All these findings are lacking in malignant sarcomas. We must point out that leiomyosarcomas of smooth muscle origin show a myxoid stroma in about $25 \%$ of cases, but the dense bundles of smooth muscle may distinguish this tumor from myxoma.

During the surgery of myxoid tumors one may suppose a myxoma is there, but histopathology unexpectedly reveals the opposite and that the mass is not myxoma but rather a myxoid form of a primary leiomyosarcoma. For such lesions, immunohistochemistry may be of great value to confirm the diagnosis (1-4). From the clinical point of view, a rapid clinical course, or multiple growths, and non-septal attachment of the mass suggest a malignant process. Careful preoperative evaluation is essential to identify such malignancies. Others stress a comprehensive preoperative workup. Here, we focus on the intra-operative observation of left atrial masses attached to lateral atrial wall. On inspection by surgeon, the malignant tumors tend to infiltrate the mitral annulus and leaflet (1-6). From the point of view of cellular proliferation, Ki67 can be used as a reliable marker to show that myxomas are slowly growing benign neoplasms, because we note that few cells (up to 5\%) stain positive with this cellular proliferative index marker (7). Malignant sarcomas of the heart show a tendency to have myxoid degeneration. Therefore, meticulous clinical and histopathological observation of suspicious cardiac tumors is deemed advisable and should be verified by further imaging examination (8). Once again it should be emphasized that histopathology and immunohistochemistry (IHC) are essential to confirm the diagnosis of such an aggressive malignant tumor (9). 


\section{References}

1. Mozaffari K. All That Glitters Is Not Gold and All Myxoid Tumors Are Not Myxomas. ; 2010.

2. Dehmeshki J, Ruto AC, Arridge S, Silver NC, Miller DH, Tofts PS. Analysis of MTR histograms in multiple sclerosis using principal components and multiple discriminant analysis. Magn Reson Med. 2001;46(3):6009. [PubMed: 11550255].

3. Chopra P, Ray R, Saxena A. Illustrated textbook of cardiovascular pathology. CRC Press; 2013.

4. Morin JE, Rahal DP, Huttner I. Myxoid leiomyosarcoma of the left atrium: a rare malignancy of the heart and its comparison with atrial myxoma. Can J Cardiol. 2001;17(3):331-6. [PubMed: 11264566].

5. Hasegawa T, Nakagawa S, Chino M, Kunihiro T, Ui S, Kimura M. Primary cardiac sarcoma mimicking benign myxoma: a case report. J Cardiol.
2002;39(6):321-5. [PubMed: 12094522].

6. Kim JT, Baek WK, Kim KH, Yoon YH, Kim DH, Lim HK. A primary cardiac sarcoma preoperatively presented as a benign left atrial myxoma. Yonsei Med J. 2003;44(3):530-3. doi: 10.3349/ymj.2003.44.3.530. [PubMed: 12833594].

7. Roskell DE, Biddolph SC. Proliferating cell nuclear antigen expression grossly over-estimates cellular proliferation in cardiac myxomas. Eur J Med Res. 1999;4(3):105-6. [PubMed:10085277].

8. Etschmann B, Krombach G, Boning A, Gattenlohner S. Pleomorphic high-grade sarcoma of the heart mimicking cardiac myxoma. BMJCase Rep. 2012;2012 doi: 10.1136/bcr.12.2011.5277. [PubMed: 22605601].

9. Aggarwal T, Goyal S, Zaheer S. Pleomorphic rhabdomyosarcoma of the left atrium mimicking myxoma. Indian J Pathol Microbiol. 2016;59(3):379-81. doi: 10.4103/0377-4929.188138. [PubMed: 27510683]. 